\title{
Getting Beyond Form Filling: The Role of Institutional Governance in Human Research Ethics
}

\author{
Gary Allen
}

Published online: 30 April 2008

(C) Springer Science + Business Media B.V. 2008

\begin{abstract}
It has become almost a truism to describe the interaction between research ethics committees and researchers as being marred by distrust and conflict. The ethical conduct of researchers is increasingly a matter of institutional concern because of the degree to which non-compliance with national standards can expose the entire institution to risk. This has transformed research ethics into what some have described as a research ethics industry. In an operational sense, there is considerable focus on modifying research behaviour through a combination of education and sanctions. The assessment of whether a researcher is 'ethical' is too often based on whether they submit their work for review by an ethics committee. However, is such an approach making a useful contribution to the actual ethical conduct of research and the protection of the interests of participants? Does a focus on ethical review minimise institutional risk? Instead it has been suggested that ethics committees may be distorting or frustrating useful research and are promoting a culture of either mindless rule following or frustrated resistance. An alternative governance approach is required. There is a need for a strong institutional focus on promoting and supporting the reflective practice of researchers through every stage of their work. By situating research ethics within the broader framework of institutional governance, this paper suggests it is possible to establish arrangements that actually facilitate excellent and ethical research.
\end{abstract}

Keywords Ethics committee - Institutional governance $\cdot$ Research ethics · Research practice $\cdot$ Rules

\section{All is Far From Quiet on the Western Front}

Too often the climate between research administrators, ethics committee members and researchers is acrimonious. It seems there is an emerging culture of distrust between researchers and research ethics committees. The regulation of ethical conduct in human research has become something of an industry (Ashcroft 1999, p14), where a proliferation

G. Allen $(\bowtie)$

Research Ethics, Griffith University, Brisbane, Australia

e-mail: gallen@ecn.net.au 
of rules has created an 'out of control bureaucracy' that often impedes the conduct of research (Haggerty 2004, pp. 392-394).

It is not difficult to find examples of researcher frustration with the behaviour of ethics committees (Furedi 2002) or questions about the sustainability of the existing ethical review systems (Komesaroff 2002). Furthermore, there are repeated suggestions that the functioning of ethics committees may actually encourage an abrogation of the responsibility of researchers to reflect upon the ethical issues associated with their own research (Loff and Black 2004; Schwartz 2000). There are also warnings that the existing approach to the ethical review of research may actually be hampering (or 'distorting') useful research (Israel 2004, p. 6; Hoonaard et al. 2004, p. 11).

Unfortunately, national research ethics frameworks can often be treated by ethics committees, administrators and institutions as though they were to be read and applied literally without a reflection on the specifics of a particular individual case. This can encourage a mindset that if an approach to an ethical dilemma is not explicitly anticipated in the national guidelines it is not permissible. It also tends to result in specific provisions of the guidelines being applied in a one-size-fits-all manner, where the realities and context of the specific project are ignored. Experience suggests that this kind of situation most frequently occurs when an over-worked ethics committee is confronted with a research design, approach or situation which is unfamiliar.

It is important to remember that, in many jurisdictions, responsibility for the conduct of researchers, and the ability to take action in response to inappropriate conduct, resides at the institutional level. An important first step in the improving the institutional climate between researchers and research ethics committees is recognising that the ethical conduct of researchers is an institutional governance issue, which should be imbedded within the institution's broader governance framework.

\section{Institutional Risk}

Concurrently with the concerns about the operation of research ethics systems, 'regulators' are placing an increasing focus on the responsibilities of institutions to ensure the ethical conduct of research conducted within the institution (Breen 2002). In many jurisdictions institutions must ensure researchers are trained in their ethical obligations. Institutions must also have systems to ensure compliance with the national standards and to actively monitor the conduct of researchers. A failure to meet these institutional responsibilities can attract serious penalties - for example all research funding ${ }^{1}$ to the institution may be suspended (for discussion about the 'Johns Hopkins case', see Abate and Russel 2001; Hotchin 2001; Keiger and de Pasquale 2002). Indeed, there are some very public examples of an entire institution being penalised for the actions of a single research team and/or because of perceived systemic failures within the institution.

This has resulted in a climate where institutions feel unable to trust researchers to 'do the right thing'. Instead, many institutions turn to a bureaucratic structure to ensure compliance with ethical and regulatory standards (ALRC and AHEC 2001, pp. 109-110; Dodds et al.

\footnotetext{
${ }^{1}$ The research funding to an institution comes from multiple sources, so any impact upon funding (e.g. from the National Health and Medical Research Council) might initially impact upon a section of research funding to the institution. However, given the degree to which multiple funding bodies require institutional compliance with the relevant national framework, there may be a cascading effect that impacts upon most, or at least a significant proportion of an institution's funding.
} 
1994, p. 19). This in turn perpetuates an adversarial tone to the interactions between researchers and ethics committees (Shaul 2002). In such a context, ethics committees are perceived as having a 'policing' role, with the objective of catching researchers in wrongdoing.

The combination of an institutional focus on compliance, with a view that researchers regard the work of HRECs with something between truculence, petulance or ignorance, appears to dominate the thinking of a majority of administrators, ethics committees and even regulators.

This is the perceived 'compliance problem' that must be tackled - largely to safeguard the institution from the serious consequences of a regulator or funding body judging the institution to be non-compliant.

The obvious limitation of this increasingly dominant way of viewing the interaction between researchers and research ethics committees is that it tends to be conceptually constraining. Within this constraint, any reflection on the role or operation of ethics committees tends to be almost exclusively focussed upon the goal of ensuring compliance, and compliance tends to be understood in terms of a researcher completing an application form for review by an ethics committee (Loff and Black 2004).

In effect, institutions perceive the 'compliance problem' to be any researcher who does not submit their work for ethical review or otherwise adhere to the institution's research ethics processes. However, in fact the 'compliance problem' might actually be the deleterious impact upon research practice when an institution relies upon directive rules and the threat of sanctions to modify researcher behaviour.

The limited perspective of focussing on ethical review as a question of compliance ignores the interesting question of why a researcher, who might otherwise ascribe to the highest ethical standards and holds a very real personal commitment to the protection of research participants, might adamantly refuse to submit his work to review by a research ethics committee. It also glosses over the problem of a researcher who dutifully submits her work to ethical review, but in fact only begrudgingly pays lip service to the principles that supposedly underpin that review. As noted by Haggerty (2004, p. 410) a schism has emerged between the actual ethical conduct in research and the mechanism of following the rules.

One of the reasons governing researcher practice can be so problematic is the realities of academic practice within a multi-disciplinary university, which can often be spread over geographically dispersed campuses. It is practically impossible for an institution to know exactly how individual researchers are conducting themselves. Given the degree to which inappropriate behaviour by even an individual researcher can expose the institution to risk, this creates understandable anxiety and a desire to try to centrally regulate conduct in a way that can be demonstrated to an external agency.

Could it be that, we have allowed the goal of demonstrable compliance to subsume the principle of facilitating excellent ethical research and protecting the rights of research participants? It seems that in our rush to minimise institutional risk and to have a 'sound' governance framework, somehow research ethics has become about expertly filling out a form for a committee, rather than an ongoing active reflection on important issues and their application to useful practice.

\section{The Typical Approach to the 'Compliance Problem'}

In many cases, the typical governance approach to the 'compliance problem', seems to be predicated on an unquestioning acceptance of what Woodward (1994) described as 
'Weberian Orthodoxy'. This approach assumes that the best way to regulate practice within an organisation is through the release of detailed centrally formulated rules that are intended to closely direct practice in an absolute way. The rapidly increasing layers of rules and expanding role for ethics committees is a manifestation of this 'Orthodoxy' (Haggerty 2004, p.394). Also implicit in this dominant approach to research ethics systems is the use of what Hart described as a sanctions and enforcement model of control - where it is assumed that if the mere existence of the rules is not enough to modify behaviour then the threat of severe penalties will be (Hart 1999).

A typical symptom of this orthodoxy, are 'research ethics educational' strategies that focus upon the correct completion of forms, the mechanism by which work is submitted for ethical review, and the textual features of informed consent materials. Often absent from such programs is much in the way of discussion about ethical conduct during the research, or resources to assist a reflective approach to the ethical challenges researchers might face.

In fairness to the typical ethics committee, it should be acknowledged that a combination of crippling workload and limited resources can makes it difficult for a committee to do anything other than review the huge volume of new applications submitted to each meeting (McNeill et al. 1990; Federman; Hanna et al. 2003, pp. 8-9). There is simply not enough resources or time to enable the committee to be proactive in other areas (Breen et al. 2005, p. 469). This overwhelming workload is often cited as the reason a HREC does not take a more active role in educational strategies or policy development (Frew 2001, p. 67).

But it could reasonably be asked, is the heavy workload of ethics committees and administrators really the source of the problem, or is it a symptom? As long as administrators and committee members continue to accept without question the notion that tackling the 'compliance problem' is a priority issue and the best way of tackling this problem is by modifying the behaviour/knowledge level of researchers through threats, 'evangelical education', or better 'form filling', then it might usefully be asked whether more time or resources would necessarily improve the climate between researchers and research ethics committees. Indeed there is a very real risk that greater resourcing for a research ethics committee's compliance/educational strategies might in fact compound the problem. An educational program based solely upon the goal of reducing compliance problems by addressing 'researcher ignorance of ethical standards', might easily be construed as extremely patronising by researchers and further poison the relationship between committees and researchers.

\section{Limitations of the Typical Approach}

There are sources of evidence available that point to limitations of the typical approach to research ethics.

Firstly, there continue to be public cases arising from breaches in ethical standards by researchers and/or failures in institutional research ethics systems (Unknown 2000; Oakes 2002 p450; Shaul 2002). Furthermore, some analyses of research practice point to a worrying level of ethical problems (Dotterweich and Garrison 1998, p. 444; Payne 2000, pp. 2-7; Thompson 1984).

Secondly, there is a significant body of commentary from researchers that point to serious concerns about the approach to the application of research ethics systems (Fitzgerald and Yule 2004, pp. 36-38; Oakes 2002 pp. 445-446; Singer 1989). 
Thirdly, there are worrying indications that research designs are being compromised or 'disfigured' by existing ethical review processes (Haggerty 2004, p. 412; Hoonaard 2004, pp. 11-12; Iphofen 2004), or that researchers may actually be presenting a distorted description of their research to get it past an ethics committee (Shore and West 2005).

Lastly, the simple fact that there is such an energetic international discussion about the sustainability of research ethics systems (Ramcharan and Cutcliffe 2001; Dodds 2002; Fitzgerald and Yule 2004) serves as indication that there is a level of institutional disquiet about the appropriateness and results of the current approach.

\section{Problems in the Breach}

It is perhaps tempting for research administrators and ethics committee members to blame any problems with the typical approach to research ethics on our respective national frameworks.

Certainly, in Australia, the current edition of the National Statement on Ethical Conduct in Research Involving Humans (NHMRC 1999) is not without its limitations and short comings. The National Statement cites biomedical texts, such as the Declaration of Helsinki as the source of its authority. At the same time the National Statement fails to acknowledge other sources of ethical guidance for researchers outside of the health sciences, such as codes of conduct for psychologists (Dodds et al. 1994, p. 16). Much of the language and specific matters addressed within the National Statement are focussed on biomedical issues, with apparent assumptions about research practice that are more relevant to quantitative approaches. Fortunately, it appears that the next edition of the National Statement will address many of these issues and be far more relevant to the practice of research outside of the health sciences.

Despite the temptation to blame our current difficulties on the relevant national guidelines, it should be acknowledged that, even when a national framework articulates principles with broad application to all human research, the way in which they can be applied at the institutional level is the source of many of the difficulties. In his report for the New South Wales Bureau of Crime Statistics and Research, Israel (2004) quotes specific cases of how the sometimes bizarre demands of an ethics committee can seriously impact upon the conduct of criminological research. This situation results in delays, considerable frustration and distrust, and can result in research being distorted to the point where it is unworkable. In these cases, the problem was not whether there was dispute over the validity of an ethical principle, but instead related to a policy position and approach adopted by an ethics committee.

My own experience during the last ten years working with ethics committees in Australia, Canada, England and Vietnam is that committee members often feel isolated from, and under appreciated by, their own institution. This isolation can lead to a sense that members are charged with "protecting the institution", but are unsure whether the institution supports them. When combined with a committee's phenomenal workload (Shaul 2002; Allen 2005, p. 3), this insecurity and isolation can often result in a committee taking what Haggerty (2004, p. 412) described as an overly cautious and conservative positions on issues - even when the national framework enables them the freedom to assess the specifics of a case and consider alternative approaches (Haggerty 2004, p. 412).

The approach to multi-site research is a good example of where 'failures' in the current approach to research ethics can occur at the institutional, rather than at the national level. 
This is an area that is a common source of criticism of human research systems. Researchers frequently complain about the inefficiencies, delays and frustrations associated with having to apply to multiple ethics committees for one study ${ }^{2}$.

This occurs in Australia despite the fact that the current edition of the National Statement urges institutions to "minimise unnecessary duplication in review of multi-centre research" and suggests an ethics committee can decide to "accept the decision of another institution, organisation or HREC in relation to multi-centre research" National Statement (1999).

Gold and Dewa (2005) point to similar problems with the processing of multi-site research in the USA. Like Australia, the American system allows for, and even actively encourages, ethics committees to accept the review decision of another committee for multi-site research (NBAC 2001, p. 122).

In each case, the problems and frustrations faced by researchers conducting work at multiple sites has very little to do with the relevant national research ethics framework, but a great deal to do with the approach at the institutional level. It seems there is an active resistance of the use of reciprocal or centralised review solutions - even when the national frameworks encourages such an approach (Lux et al. 2000, p. 1183).

When discussing this issue with ethic committee members and administrators I have frequently heard comments justifying this kind of resistance on the basis of the committee's duty of care, responsibility and culpability. Such comments are often wrapped in deceptively compelling references to the law and insurance. Yet the implicit principles that underpin such arguments rarely seem to have been tested with the institution's legal area. Furthermore, as noted above, any refusal to engage in some form of reciprocal or fasttracked review is contrary to the specific provisions of the relevant national framework.

An institution involved in a multi-site project, does indeed have its own governance responsibilities for that research -including a duty of care to staff, students or patients. However, the presence of such responsibilities does not create a requirement for review by a research ethics committee. It does require that the institution have appropriate governance systems to ensure that the risks and issues are appropriately assessed and addressed.

Rather than leaving ethics committees to struggle with the question of their responsibility, duty of care and accountability, there is a need for an institutional-level policy decision about how to handle multi-site research, including issues such as reciprocity.

\section{Facilitation and Legitimacy as a New Theoretical Model for Governance Systems}

If we are to tackle the goals of managing institutional risk, supporting ethical conduct in research, and protecting the interests of research participants then what is required is a complete departure from the existing approach to research ethics.

No longer should we allow research ethics, and research ethics committees to operate independently of an institution's broad approach to research governance. No longer should we allow 'doing research ethics' to mean filling out a form and interacting with an overworked committee that is struggling to work out its place within the institution.

For local research ethics arrangements to have any hope of having a positive impact upon the design and conduct of research, they must first be predicated on the goal of facilitating excellent and ethical research.

\footnotetext{
${ }^{2}$ For example, in 2005 this matter received considerable attention during the plenary and concurrent sessions of both the NHMRC conference on human research ethics and the annual conference of the Australasian Research Managers Society.
} 
I suspect that very few research administrators or research ethics committee members would disagree that facilitating research is important. Indeed, in Australia the National Statement (NHMRC 1999, p. 1) specifies that, in addition to its primary purpose of safeguarding the rights and welfare of participants, "an important secondary purpose" is to facilitate research. However, ethics committee members and administrators need to embrace facilitation as a core element of the operation of an institutional research ethics system. Rather than trying to catch researchers in wrongdoing or 'educate them in ethics', the whole focus of the system becomes a collaborative exchange between the stakeholders.

Of course, that is not to imply that the primary objective of safeguarding the welfare and rights of research participants should in any way be compromised. Embracing the tenet of facilitation just means that the various players in the system try to work collaboratively with researchers, rather than try to 'police' behaviour. In such an approach, there is an attempt to build an equal partnership between researchers and the ethics committee.

In her book on trust in society Misztal (1996, pp. 26-27) discussed how trust plays an important role in the support for, and participation in, public policy. Misztal (1996, pp. 245-269) suggested that the degree to which individuals see any exercise of public power as legitimate is a fundamental factor in determining whether they will comply with that power. I would suggest that a strength of this perspective on the interaction between instruments of regulatory control and the behaviour of practitioners is that it recognises the realities of decision making and practice in what Lash (1994, pp. 208-209) referred to as post-modern organizations (Lash 1994, pp. 208-209). For example - researchers have a degree of autonomy, they operate within fluid working arrangements and teams, and face a dizzying array of challenges that defy any directive-rule-based attempt to regulate their practice. It is simply disingenuous to assume that a central prescriptive rule can offer applicable solutions to the ethical challenges that researcher will face.

We need to recognise that, especially for researchers in some disciplines, there may be perceived legitimacy problems with the national research ethics framework. Consequently, institutions have an important role to play in filling this legitimacy gap through the implementation of local processes and practices that can be perceived as being more relevant and applicable to the practice of researchers within the institution.

Rather than trying to use directive rules to prescribe conduct, an institution's research ethics arrangements need to encourage reflective practice where the researcher has an active role in formulating, and justifying, the solution to the issue - not just at the point of seeking ethical clearance, but throughout the entire life of a research project.

\section{The Griffith Model}

Griffith University is a multi-campus university based in the southeast corner of Queensland, Australia. It is a vibrant and growing institution, within what is called the Innovative Research Universities Australia group.

The situation in terms of the approach to human research ethics at Griffith University, prior to 2003, was not that different from the problems and difficulties faced by many other Australian universities. For a variety of historical and structural reasons, research ethics had become isolated from the University's broader research governance framework. This situation compounded the tendency of the ethics committee to take a cautious and conservative approach to risks and unfamiliar research designs. Furthermore, an overwhelming workload and insufficient resourcing meant that much of the University's 
guidelines had not been updated for a number of years and predate the release of the current national research ethics framework.

The negative impact of this situation was most obviously discernible in three ways: (1) The Director, Office for Research, the Deputy Vice-Chancellor (Research), and even the Vice-Chancellor, were receiving repeated complaints from senior researchers about "the ethics problem"; (2) even though there was a continual growth in the amount of research ethics activity, this was largely concentrated into a handful of broadly health-related disciplinary areas; and (3) there was increasingly an adversarial, even besieged, tone to the relationship between the HREC and the research community.

In 2003, the then Deputy Vice-Chancellor (Research) commissioned a major review of human research ethics at Griffith University. The report of the Research Ethics Review 2003 was endorsed at the executive level. This provided top level support for a reform agenda.

A new model for the governance of ethical conduct of human research arose from this review. The key features of this new model are outlined below.

Executive Officer Support The University created a new senior policy officer position, at equivalent to senior lecturer level. Separate from the traditional secretariat support for the ethics committee, this position performs an advisory, policy development and educational role. Furthermore, there has been a limited 'buy-out of the Chair and Deputy Chairperson of the ethics committee.

Research Ethics Advisor network Every academic element of the University has appointed one member of academic staff as a Research Ethics Advisor (REA) for their area. REAs undertake a number of roles including: providing local 'judgement free' and discipline relevant advice to students, supervisors and other researchers; delivering workshops in their area; participating in the expedited review system (see below); advising the head of the element on research ethics matters; and facilitating communication to and from the committee.

Proportional Ethical Review The University now has three ethical review pathways, which tie the amount of paperwork, the rigour of the review; and the processing time to a smart assessment of the risks and ethical issues associated with the proposed work. Two of these review pathways are online, with an online tool to assist the applicant accurately determine the appropriate review pathway.

Research Ethics Manual The University has produced an online, booklet-based manual for researchers. Each booklet is focussed on a particular ethical issue or research design (such as online research). Even though the manual does outline external regulatory requirements and approaches previously accepted by the ethics committee, these are not presented as directive rules. Instead the Manual is intended to inform a researcher's understanding of the principles and to assist them to justify alternative approaches.

Case Study Training The approach to research ethics training within the University is not based solely on the process of ethical review and better 'form filling'. Instead, real cases are used to explore issues that can be raised during the design, ethical review, conduct and publishing of human research. This approach to training stresses a two-way learning process, where the experience of researchers is fed back into the institutional approach to governance. 
Targeted Arrangements The University has adopted special policies and processes for research undertaken by coursework and undergraduate students, multisite research and research involving 'standard' instruments. There are also supplementary processes for research involving Indigenous Australians, clinical trials, and research conducted overseas.

\section{The Griffith Experience: 2 Years On}

At the time of writing, the 'Griffith Model' has been operating for just over 2 years. To date, there has not been any systematic collection of data about the impacts of the 'Griffith Model'. However, there are some sources of data and testimony that make it possible to at least suggest the degree to which the changes to governance at Griffith University have had a significant and enduring positive impact.

\section{Attitudes Towards Research Ethics}

In 2005, the Office for Research at Griffith University was subject to a comprehensive external review by a panel of executive-level academics and senior research administrators. The review process involved the review panel meeting with a range of researchers and key stakeholders, as well as considering confidential submissions from across the University.

In their written report noted (Glover et al. 2005):

- That the institution's research community showed significant support for the changes to the University human research ethics governance arrangements (p. 17);

- That there was "uniformly strong endorsement" for the University's research ethics system, which was described as "effective and responsive" (p. 8); and

- That the work and contribution of the new ethics team was greatly appreciated (p. 8).

Clearly this represents a significant turnaround in climate at Griffith University in only a few short years. This major change in climate is also reflected in my anecdotal observations and in the feedback that I continue to receive from researchers and support staff.

\section{Participation Rate}

In 2004/2005 a total of 559 applications for ethical review were submitted at Griffith University, as opposed to 430 applications in 2003/2004, representing a $30 \%$ increase in activity. This followed a $40 \%$ increase in activity $2003 / 2004$, compared to $2002 / 2003$. The significant increase in new applications in 2004/2005 came at the same time as there was a $150 \%$ increase in variations to approved projects. As part of the 'Griffith Model', new processes were implemented to make it easier for a research team to extend and vary an approved protocol to cover the next phase of a research project. In practice a variation can often negate the need for a research team to submit a new ethics application for a new phase of previously approved research.

It is also worthwhile commenting on how the above activity is now spread across individual University elements. During the period 2003-2005 some areas of the University have seen massive increases in activity, such as: Environmental Planning; Film, Media and Culture; Hospitality, Sport and Leisure; Information Computer Technology; Marketing; and 
the Queensland Conservatorium of Music. These are areas that traditionally had relatively low levels of human research ethics activity.

To some degree, these increases in activity can be attributed to the maturing research profile of Griffith University. However, clearly increases of the order reported above are not reflected in improvements in the University's grant success rates, publication rates and research higher degree student completion rates. I would suggest that, to a large degree, the massive jump in activity reflects a surge in researcher participation in, and engagement with, the University's governance framework. I would contend that the work to address the 'legitimacy gap' of the national framework and previous University arrangements has resulted in not only more researchers electing to participate in the framework, but also a more complete spread of this activity into disciplinary areas outside of the health sciences.

\section{Client Service, Efficiency and Workload}

In 2004/2005 almost 90\% of Griffith University's human research ethics activity was reviewed via one of the two expedited review pathways (Allen 2005, p. 5). Coupled with the variation mechanism, course clearances, approved protocol procedure and multi-site arrangements this made a considerable contribution to the way in which the needs of researchers (the clients of many of the systems) are met. In many cases, requests from researchers were resolved in a matter of a few days.

The changes outlined to the University's governance framework also generated considerable administrative and committee efficiencies. For example, rather than the ethics committee struggling through in excess of 30 applications at each of its monthly meetings, the committee now reviews an average of five applications. Rather than making 20+ copies of 30 applications, and posting these to each member, Office for Research needs only expend a fraction of the resources to support the new multi-pathway review process. A significant impact of this change is that the ethics committee now has time to actively reflect upon policy issues and discuss emergent issues. The committee can also now afford to take the time to carefully reflect upon the ethically sensitive research that is submitted for full review.

It is unlikely that the previous University systems, resources and arrangements could have coped with the recent increases in workload. Even the modest growth in activity seen in the years prior to 2003 would probably have made the HREC's workload unsustainable. During this period the University has established a new medical school, and commenced strategic collaborations with bodies such as the Queensland Institute of Medical Research. Despite the extra volume and complexity arising from these institutional developments they have not placed any significant strain on the governance framework or the HREC, because of the reforms commenced in 2003.

Nevertheless, it would be extremely short-sighted to believe that further work is not needed at Griffith University, or the challenges ahead will not demand further innovation.

\section{Conclusion}

The typical approach to research ethics is neither successful or desirable. In many cases it generates a culture of distrust and resentment between researchers and research ethics committees. At worst, the typical approach may be distorting or blocking useful research, 
without necessarily having a positive impact upon research practice or the interests of research participants.

Institutions have an important role to play, by establishing local arrangements that address any legitimacy gap between the relevant national research ethics framework and research practice. The focus of these arrangements needs to be on fostering and supporting reflective practice, not unquestioning rule following.

The experience at Griffith University suggests that it is possible to have a significant impact upon the climate between researchers and ethics committees, improve client service and efficiency, and achieve better outcomes, from the adoption of an approach that treats research ethics as an institutional governance issue.

\section{References}

Abate, T., \& Russel, S. (2001). Shutdown puts spotlight on human research Experts say Johns Hopkins case reflects problems across U.S. Retrieved 7 Mar 2002 from http://www.sfgate.com/cgi-.

Allen, G. (2005). Griffith University research ethics advisor system. HREC Bulletin, Summer, 9.

Allen, G. (2005). Griffith University HREC Annual Report 2004/05. Brisbane: Griffith University.

ALRC, \& AHEC. (2001). Protection of Human Genetic Information: Issues Paper. Canberra: Australian Law Reform Commission.

Ashcroft, R. E. (1999). The new national statement on ethical conduct in research involving humans: A social theoretic perspective. Monash Bioethics Review, 18(4), 14-17 (Ethics Committee Supplement).

Breen, K. (2002). Improving Australia's ethical review processes - Slow and steady wins the race. Monash Bioethics Review, 21(3), 58-62.

Breen, K., McNeil, J., \& Walsh, M. (2005). Improving the governance of health research. The Medical Journal of Australia, 182(9), 468-471.

Dodds, S., Albury, R., \& Thomson, C. (1994). Ethical research and ethics committee review of social and behavioural research proposals: report to the Department of Human Services and Health. Canberra, ACT: Dept. of Human Services.

Dodds, S. (2002). Is the Australian HREC system sustainable? Monash Bioethics Review, 21(3), 43-48.

Dotterweich, D. P., \& Garrison, S. (1998). Research ethics of business academic researchers at AACSB institutions. Teaching Business Ethics, 1, 431-447.

Federman, D. D., Hanna, K. E., \& Rodriguez, L. L. (2003). Responsible research: A systems approach to protecting research participants. Washington, DC: The National Academies Press.

Fitzgerald, M. H., \& Yule, E. (2004). Open and closed committees. Monash Bioethics Review, 23(2), 35-49.

Frew, R. (2001). Research ethics committees: What can we learn from the Western European and United States experience. Monash Bioethics Review, 20(2), 61-77.

Furedi, F. (2002). Don't rock the research boat. The Times, January 11.

Glover, B., McBriarty, L., Dibb-Smith, J., Johnstone, R., Thiel, D., \& Clifford, R. (2005). Report of the panel reviewing Office for Research, Griffith University.

Gold, J. L., \& Dewa, C. S. (2005). Institutional Review Boards and Multisite Studies in Health Services Research: Is there a better way? Health Services Research, 40(1), 291-308. DOI 10.1111/j.14756773.2005.00354.x.

Haggerty, K. D. (2004). Ethics creep: Governing social science research in the name of ethics. Qualitative Sociology, 27(4), 391-414.

Hart, H. L. A. (1999). The elements of law(Article 1961). Retrieved 15 Mar 2002 from http://www.kentlaw. edu/classes/rwarner/justice/syllabus/helement.html.

Hoonaard, W. v. d., Given, L., Lévy, J., McGinn, M., O'Neill, P., Palys, T., et al. (2004). Giving voice to the spectrum: Report of the Social Sciences and Humanities Working Committee. Ontario: Interagency Advisory Panel on Research Ethics.

Hotchin, S. (2001). Johns Hopkins'studies involving human subjects suspended following healthy volunteer's death. Retrieved 7 Mar 2002 from http://www.sonoma.edu/users/n/nolan/n400/johnshopkins.htm.

Iphofen, R. (2004). A code to keep away judges and MPs. The Times, January 16.

Israel, M. (2004). Ethics and the Governance of Criminological Research in Australia. Sydney: Report for the New South Wales Bureau of Crime Statistics and Research.

Keiger, D., \& De Pasquale, S. (2002). Trials \& tribulation. Johns Hopkins Magazine, February 2002. 
Komesaroff, P. A. (2002). Response to Susan Dodds: Is the Australian HREC system sustainable. Monash Bioethics Review, 21(3), 68-71.

Lash, S. (1994). Expert-systems or situated interpretation culture and institutions in disorganised culture. In U. Beck, A. Giddens \& S. Lash (Eds.), Reflexive modernisation. Oxford: Polity.

Loff, B., \& Black, J. (2004). Research ethics committees: what is their contribution. Medical Journal of Australia, 181(8), 440-441.

Lux, A. L., Edwards, S. W., \& Osborne, J. P. (2000). Responses of local research ethics committees to a study with approval from a multicentre research ethics committee. British Medical Journal, 320(7243), 1182-1183.

McNeill, P. M., Berglund, C. A., \& Webster, I. W. (1990). Reviewing the reviewers: a survey of institutional ethics committees in Australia. The Medical Journal of Australia, 152, 289-296.

Misztal, B., A. (1996). Trust in modern societies. Cambridge: Polity.

NBAC (2001). Ethical and policy issues in research involving human participants. Maryland: National Bioethics Advisory Commission.

NHMRC. (1999). National statement on ethical conduct in research involving humans. Canberra: Australian Government.

Oakes, M. (2002). Risks and wrongs in social science research. Evaluation Review, 26(5), 443-479.

Payne, S. L. (2000). Challenges for research ethics and moral knowledge construction in the applied social sciences. Journal of Business Ethics, 26(4), 307-318.

Ramcharan, P., \& Cutcliffe, J. R. (2001). Judging the ethics of qualitative research: considering the 'ethics as process' model. Health and Social Care in the Community, 9(6), 358-366.

Schwartz, M. (2000). Why ethical codes constitute an unconscionable regression. Journal of Business Ethics, 23, 173-184.

Shaul, R. Z. (2002). Reviewing the reviewers: the vague accountability of research ethics committees. Critical Care, 6(2), 121-122.

Shore, N., \& West, P. (2005). Social work researchers and the Institutional Review Board: Benefits, Challenges, and Ideas for Support. Journal of Social Work Values and Ethics, 2(2).

Singer, P. (1989, 14 Nov 1989). Rats, patients and people: Issues in the ethical regulation of research. Paper presented at the Annual lecture, Coombs Lecture Theatre, Australian National University.

Thompson, W. C. (1984). Research on human judgement and decision making: implications for informed consent and institutional review. In B. H. Stanley, J. E. Sieber, \& G. B. Melton (Eds.) Research ethics: A psychological approach (pp. 37-72). London: University Nebraska Press.

Unknown, M. (2000). News in brief: Research suspensions. Monash Bioethics Review, 19(2), 1 (Ethics Committee Supplement).

Woodward, A. (1994). Making ethics part of real work. In N. Preston (Ed.)Ethics for the public sector: Education and Training (pp. 219-236). Leichardt: The Federation Press. 